\title{
Potencial evocado auditivo de tronco encefálico: estudo da via aérea e da via óssea em lactentes com alterações de orelha média
}

\section{Auditory brainstem response: study of the air and bone conduction in infants with middle ear changes}

Priscila Karla Santana Tomita ${ }^{1}$ (D), Marisa Frasson de Azevedo ${ }^{1}$ (D)

\begin{abstract}
RESUMO
Objetivo: Comparar os limiares eletrofisiológicos do potencial evocado auditivo de tronco encefálico por frequência específica (PEATE-FE) por via aérea (VA) e via óssea (VO), em lactentes com e sem alteração condutiva, verificando a aplicabilidade do gap aéreo-ósseo no diagnóstico diferencial dos tipos de perda auditiva e sua correlação com os resultados da timpanometria e do PEATE clique. Método: Amostra de 73 lactentes: grupo estudo (52 orelhas), com alteração condutiva, emissões ausentes, otoscopia e curva timpanométrica alteradas, e grupo controle ( 82 orelhas normais). O PEATE clique foi realizado a $80 \mathrm{dBNA}$ e a pesquisa do limiar (clique, 500 e $2000 \mathrm{~Hz}$ ) foi registrada por condução aérea e óssea. A timpanometria foi obtida com sonda de $1000 \mathrm{~Hz}$ (crianças até 6 meses) e de $226 \mathrm{~Hz}$ (maiores de 6 meses). Resultados: $\mathrm{O}$ grupo estudo apresentou limiares por via aérea aumentados e via óssea normal com gap aéreo-ósseo. O grupo controle apresentou limiares normais na condução aérea e óssea sem gap. Em ambos os grupos, os limiares por via aérea em $500 \mathrm{~Hz}$ foram mais elevados e, na condução óssea, os limiares em $500 \mathrm{~Hz}$ inferiores aos de $2000 \mathrm{~Hz}$. Houve associação entre gap, curva timpanométrica e aumento da latência da onda I. Conclusão: O PEATE-FE VO pode ser considerado viável na prática clínica de um serviço público de saúde, que nem sempre dispõe de sedação. As associações da timpanometria alterada e da latência prolongada da onda I com gap aéreo ósseo reforçam a importância clínica da combinação do PEATE VA e VO por frequência específica como uma alternativa viável e eficaz no diagnóstico das perdas auditivas condutivas em lactentes.
\end{abstract}

Palavras-chave: Potenciais evocados auditivos do tronco encefálico; Lactente; Condução óssea; Testes auditivos; Perda auditiva condutiva

\begin{abstract}
Purpose: To compare the electrophysiological thresholds of the FS- ABR by air and bone conduction in infants with and without middle ear alterations, verifying the applicability of the air-bone gap in the differential diagnosis of the types of hearing loss and its relationship with the results of tympanometry and of the ABR click. Methods: The sample was composed of 73 infants: study group (52 ears) with conductive alteration, absence of emissions, otoscopy and altered tympanometric curve, and the control group ( 82 normal ears). Click $\mathrm{ABR}$ was performed at $80 \mathrm{~dB} \mathrm{nHL}$ and threshold research (click, 500 and $2000 \mathrm{~Hz}$ ) was recorded by air and bone conduction. Tympanometry was obtained with a $1000 \mathrm{~Hz}$ probe (children up to six months of age) and a $226 \mathrm{~Hz}$ probe (older than six months). Results: The control group had normal thresholds in air and bone conduction without gap. In both groups, air thresholds at $500 \mathrm{~Hz}$ were higher and in bone conduction thresholds at $500 \mathrm{~Hz}$ lower than at $2000 \mathrm{~Hz}$. There was an association between gap, tympanometric curve and increased latency of wave I. Conclusion: The FS-ABR BC can be considered a viable in the clinical practice of a public health service, which does not always have sedation. The associations of altered tympanometry and prolonged latency of wave I with air bone gap reinforce the clinical importance of the combination of $\mathrm{AC}$ and $\mathrm{BC} \mathrm{ABR}$ by specific frequency as a viable and effective alternative in the diagnosis of conductive hearing loss in infants.
\end{abstract}

Keywords: Brainstem auditory evoked potentials; Infant; Bone conduction; Auditory tests; Conductive hearing loss

\footnotetext{
Trabalho realizado na Universidade Federal de São Paulo - UNIFESP - São Paulo (SP), Brasil.

${ }^{1}$ Departamento de Fonoaudiologia, Universidade Federal de São Paulo - UNIFESP - São Paulo (SP), Brasil.

Conflito de interesses: Não.

Contribuição dos autores: PKST foi responsável pela elaboração do projeto, coleta e tabulação dos dados, delineamento do estudo, execução do projeto e elaboração do manuscrito; MFA foi responsável pelo projeto, delineamento do estudo, orientação e revisão do manuscrito.

Financiamento: Nada a declarar.

Autor correspondente: Priscila Karla Santana Tomita. E-mail: priksp@yahoo.com.br

Recebido: Maio 14, 2021; Aceito: Outubro 25, 2021
} 


\section{INTRODUÇÃO}

Nos programas de triagem auditiva neonatal, pouca importância tem sido atribuída às alterações de orelha média, por serem transitórias. Entretanto, as alterações decorrentes de um comprometimento condutivo podem comprometer a percepção da fala e dificultar a compreensão, principalmente em ambiente ruidoso, o que prejudica o desenvolvimento da linguagem da criança.

A flutuação da audição causada por alterações condutivas nos primeiros anos de vida pode prejudicar o processamento auditivo, interferindo no aprendizado escolar ${ }^{(1)}$. Nas últimas décadas, estudos dedicaram-se a discutir a seleção dos procedimentos mais apropriados para avaliar neonatos e lactentes ${ }^{(2,3)}$.

As emissões otoacústicas e as medidas de imitância acústica são medidas objetivas que contribuem para a obtenção de informações importantes para avaliar as condições da cóclea e da orelha média, porém, não detectam o grau da perda auditiva.

O potencial evocado auditivo de tronco encefálico (PEATE) é recomendado ${ }^{(4)}$ como padrão -ouro para auxiliar no diagnóstico precoce das alterações auditivas na população infantil, pois permite detectar o tipo e o grau da perda.

A pesquisa do PEATE por frequência específica (PEATE-FE) é recomendada para determinar os limiares eletrofisiológicos nas diversas frequências, estabelecendo o grau e a configuração da perda auditiva para melhor adequar o processo de habilitação auditiva ${ }^{(3)}$.

Nos casos das alterações de orelha média, o PEATE realizado com estímulo clique pode não apresentar atraso nas latências das ondas que, associado a limiares eletrofisiológicos elevados, leva à interpretação errônea de perda auditiva sensorioneural ${ }^{(2)}$. Nesses casos, a obtenção do limiar por via óssea (VO) é o procedimento mais indicado para realizar o diagnóstico diferencial do tipo de perda auditiva ${ }^{(5)}$, pois, nas alterações de orelha média, o PEATE por via óssea apresentará presença da onda $\mathrm{V}$ em intensidades normais, caracterizando o gap aéreo-ósseo ${ }^{(2)}$.

$\mathrm{Na}$ literatura, ainda há controvérsia sobre protocolos de PEATE-FE por via óssea, o que dificulta o diagnóstico diferencial entre a perda condutiva e sensorioneural ${ }^{(2)} \mathrm{e}$ a estimativa do grau da perda e, consequentemente, a aplicabilidade clínica desse método. Nos protocolos canadenses ${ }^{(6,7)}$, a via óssea é recomendada quando os limiares estão elevados por via aérea (VA), sendo realizada nas frequências de $500 \mathrm{~Hz}$ e $2000 \mathrm{~Hz}$. Já o protocolo britânico ${ }^{(8)}$, recomenda a pesquisa em $1000 \mathrm{~Hz}$ e $4000 \mathrm{~Hz}$.

Além disso, o PEATE por condução óssea é utilizado em razão de fatores interferentes, como presença de $\operatorname{artefatos}^{(9)}$, necessidade, ou não, do mascaramento, uso de equipamento de um ou dois canais para interpretação dos traçados, além do modo como o vibrador ósseo é fixado e posicionado na criança.

Entretanto, há o consenso de que sua utilização no topodiagnóstico das perdas auditivas em lactentes é imprescindível ${ }^{(10,11)}$.

$\mathrm{O}$ atual estudo pretendeu contribuir para a obtenção de um diagnóstico mais preciso da perda auditiva condutiva em lactentes, além de acrescentar dados sobre a importância e a viabilidade do uso do PEATE-FE por via óssea nessa população.

O trabalho teve por objetivo comparar os limiares eletrofisiológicos do PEATE-FE por via aérea e via óssea em lactentes com e sem alteração de orelha média, verificando a aplicabilidade do gap aéreo-ósseo no diagnóstico diferencial dos tipos de perda auditiva e sua correlação com os resultados da timpanometria e do PEATE clique.

\section{MÉTODO}

Estudo transversal e observacional desenvolvido na Universidade Federal de São Paulo após aprovação pelo Comitê de Ética em Pesquisa (processo ${ }^{\circ}$ 51497). Os responsáveis pelos participantes consentiram com a realização desta pesquisa e a divulgação dos resultados, por meio da autorização e assinatura do Termo de Consentimento Livre e Esclarecido. A amostra foi composta por 73 lactentes de 1 a 12 meses, provenientes de programas de triagem auditiva neonatal e do ambulatório de diagnóstico audiológico da Instituição. Todos os lactentes realizaram triagem auditiva neonatal com menos de 1 mês de vida, e os que falharam no reteste retornaram ao ambulatório com 3 meses de idade, em média. Os lactentes que passaram na triagem auditiva foram convidados a participar do estudo e os agendamentos realizados de acordo com a disponibilidade dos pais. Os bebês foram distribuídos em dois grupos, em um total de 134 orelhas avaliadas, considerando que alguns deles acordaram e não completaram a avaliação. No grupo estudo, foram incluídos os resultados obtidos em 52 orelhas de crianças com diagnóstico médico de comprometimento condutivo, caracterizado por otoscopia alterada, curva timpanométrica $\mathrm{B}$ ou $\mathrm{C}^{(12)}$, ausência de emissões otoacústicas evocadas por estímulo transiente e PEATE clique em $80 \mathrm{dBnNA}$, sem alteração central. No grupo controle, foram incluídas 82 orelhas com presença de emissões otoacústicas, curva timpanométrica tipo A, otoscopia normal, PEATE clique normal a $80 \mathrm{dBnNA}$ e presença da onda $\mathrm{V}$ a $30 \mathrm{dBnNA}$. Excluíram-se crianças com alterações cocleares e retrococleares e/ou presença de síndromes e malformações.

As crianças foram avaliadas em sono natural, em sala com isolamento elétrico e acústico. Para o registro do PEATE clique e do PEATE-FE, utilizou-se o equipamento Smart EP, de um canal, disponível na Instituição. O preparo da pele foi realizado com pasta abrasiva. Foram usados eletrodos ECG Conductive Adhesive Medi-Trace, posicionados em Fpz (ativo), M1/M2 mastoides ipsilateral (referência) e contralateral (terra) à orelha estimulada, com impedâncias inferiores a $5 \Omega$ e diferenças intereletrodos abaixo de $3 \Omega$.

Para verificar a integridade neurofisiológica da via auditiva, utilizou-se o PEATE com estímulo clique a $80 \mathrm{dBnNA}$, sendo considerado normal o registro das ondas I, III e V com latências absolutas e valores dos intervalos interpicos I-III, III-V e I-V dentro dos padrões de normalidade para a idade cronológica. Por outro lado, no aumento das latências absolutas das ondas I, III e V com valores das latências interpicos I-III, III-V e I-V dentro do padrão de normalidade, o exame foi considerado sugestivo de alteração condutiva.

No PEATE clique foi utilizada taxa de apresentação 27,7/s, polaridade rarefeita, janela de análise de $25 \mathrm{~ms}$ e filtro passa -banda 30-1 $500 \mathrm{~Hz}$.

A pesquisa dos limiares com clique foi obtida decrescendo-se a intensidade progressivamente até encontrar a menor intensidade em que a onda $\mathrm{V}$ pôde ser observada, conforme recomendado na literatura ${ }^{(13)}$. Realizou-se a pesquisa dos limiares com tone burst nas frequências de $500 \mathrm{~Hz}$ e $2000 \mathrm{~Hz}$, utilizando-se fones de inserção ER 3A para a pesquisa da via aérea e vibrador ósseo B71 para o registro da via óssea ${ }^{(14)}$. O vibrador foi posicionado na região do osso temporal na posição superoposterior 
auricular, próximo à orelha estimulada, e mantido na posição sem o uso de banda elástica, com fixação manual realizada pelo examinador ${ }^{(15)}$. Para a pesquisa da via óssea, foi utilizado mascaramento (ruído branco) de $60 \mathrm{dBNPS}^{(16)}$, contralateral à orelha estimulada, em razão de o equipamento utilizado dispor apenas de um canal, impossibilitando a comparação dos registros ipsi e contralateral para confirmação do lado da resposta no qual a onda $\mathrm{V}$ apresentaria maior amplitude e menor latência no canal ipsilateral à cóclea estimulada ${ }^{(6)}$.

A polaridade do estímulo foi alternada com taxa de estimulação de $39,1 / \mathrm{s}$, janela de 25,6 ms, envelope blackman, filtro de 30 a $1500 \mathrm{~Hz}$ e 1000 a 3000 estímulos. O tempo de duração do estímulo na frequência de $2000 \mathrm{~Hz}$ foi de $4 \mathrm{~ms}$ e, na frequência de $500 \mathrm{~Hz}$, foi de $8 \mathrm{~ms}$, com dois ciclos de subida, 0 de platô e dois ciclos de descida. A calibração biológica foi realizada em dez adultos jovens com limiares audiométricos iguais ou inferiores a $20 \mathrm{dBnNA}$ em 500 e $2000 \mathrm{~Hz}$ por via óssea, conforme recomendação da literatura ${ }^{(6)}$. O valor de referência de $0 \mathrm{dBnNA}$ foi 36,2 dBpNPS para $500 \mathrm{~Hz}$, e 28,2 dBpNPS para $2000 \mathrm{~Hz}$.

Para diminuir a interferência eletroacústica no exame, a criança foi posicionada no colo da mãe e avaliada após a mamada, em sono natural.

A detecção da resposta baseou-se na análise subjetiva, com detecção manual das ondas e padrões de referência: função latência/intensidade, reprodutibilidade visual, ruído residual inferior a $0,08 \mu \mathrm{V}$ e relação sinal/ruído superior a $1,0^{(6)}$.

No grupo estudo, os estímulos por via aérea foram apresentados de forma decrescente, iniciando-se em 70 dBnNA. No grupo controle, tanto por via aérea, como por via óssea, o exame foi iniciado com as intensidades padronizadas como critério de normalidade, segundo a literatura ${ }^{(6)}$ : para a via área 30 dBnNA em $2000 \mathrm{~Hz}$ e 35 dBnNA em $500 \mathrm{~Hz}$ e, para a via óssea, 30 dBnNA em $2000 \mathrm{~Hz}$ e 20 dBnNA em $500 \mathrm{~Hz}$, com a finalidade de reduzir o tempo do exame. Na ausência de resposta em 10 dBnNA (mínima intensidade pesquisada), a intensidade foi aumentada de 5 em 5 dBnNA. Foi considerado o limiar eletrofisiológico com a menor intensidade, na qual a onda $\mathrm{V}$ pôde ser identificada e reproduzida, com ausência de resposta na intensidade $5 \mathrm{~dB}$ abaixo do limiar identificado ${ }^{(2)}$.

O tempo do exame foi cronometrado pelo relógio do próprio microcomputador, em tempo absoluto, desconsiderando-se as pausas para controle das condições do exame e/ou estado do lactente.

Para avaliar a mobilidade do conjunto tímpano-ossicular, a timpanometria foi realizada com o equipamento Impedance Audiometer AT235h-Interacoustics, com sonda de $1000 \mathrm{~Hz}$ (para crianças até 6 meses de idade) e de $226 \mathrm{~Hz}$ (para crianças maiores de 6 meses). Obteve-se o registro das curvas timpanométricas com variação de pressão de $+200 \mathrm{daPa}$ a $-200 \mathrm{daPa}$. As curvas timpanométricas foram classificadas em: normais: tipo A (pico de admitância entre 0,3 e 1,65 ml e pressão entre -100 e +100 daPa), com pico único ou duplo pico, e alteradas: tipo B (curva plana sem pico de admitância) e tipo C (pico deslocado para pressão negativa além de $-100 \mathrm{daPa})^{(12)}$. Para as crianças que já vieram com o diagnóstico médico de alteração de orelha média, o exame só foi realizado para confirmar a presença de curva $\mathrm{B}$ ou $\mathrm{C}$. As crianças que não vieram encaminhadas do otorrinolaringologista (ORL) e tiveram alteração no teste foram encaminhadas para ORL para confirmar a alteração condutiva e tratamento.

A análise dos dados foi inferencial aplicando os testes: Qui-quadrado, Kruskal-Wallis, Teste Z, Mann-Whitney, Teste Exato de Fisher e Coeficiente de Correlação de Spearman. O nível de significância adotado foi 5\%.

\section{RESULTADOS}

A faixa etária dos lactentes variou de 1 a 12 meses, sendo a média da idade de 3,2 meses e o tempo médio para a realização do exame de 131,7 minutos, sem diferença entre as medianas de idade e tempo do exame entre os grupos, o que significa que a variação de idade, neste estudo, não influenciou no tempo do exame. A análise dos limiares eletrofisiológicos, em decorrência da frequência sonora, demonstrou que o limiar na frequência de $500 \mathrm{~Hz}$ foi maior que $2000 \mathrm{~Hz}$ por via aérea e menor por via óssea, com diferença significativa em ambos os grupos (Tabela 1).

Em relação à comparação entre os grupos, os limiares por via aérea foram mais elevados no grupo estudo, sendo que, em $500 \mathrm{~Hz}$, essa diferença foi maior. Os limiares por via óssea

Tabela 1. Valores médios dos limiares eletrofisiológicos em dB nNA por via aérea e via óssea nas frequências de $500 \mathrm{~Hz}$ e $2000 \mathrm{~Hz}$ no grupo estudo e grupo controle

\begin{tabular}{|c|c|c|c|c|c|c|c|}
\hline Variável & $\mathbf{N}$ & Média & Mediana & $\begin{array}{l}\text { Desvio } \\
\text { padrão }\end{array}$ & Mínimo & Máximo & Valor de $p$ \\
\hline Grupo controle & & & & & & & Teste Z \\
\hline Limiar VA- $500 \mathrm{~Hz}$ & 82 & 26,6 & 30 & 5,8 & 5 & 35 & - \\
\hline Limiar VA- $2000 \mathrm{~Hz}$ & 82 & 21,5 & 20 & 7 & 5 & 35 & \\
\hline Diferença 500 X $2000(\mathrm{~Hz})$ & 82 & 5,2 & 5 & 7,4 & -20 & 25 & $<0,001^{*}$ \\
\hline Limiar VO - $500 \mathrm{~Hz}$ & 82 & 16,3 & 20 & 5,1 & 5 & 25 & \\
\hline Limiar VO - $2000 \mathrm{~Hz}$ & 82 & 21,3 & 20 & 7,1 & 5 & 30 & \\
\hline Diferença 500 X $2000(\mathrm{~Hz})$ & 82 & -5 & -5 & 6,5 & -20 & 10 & $<0,001^{*}$ \\
\hline Grupo estudo & & & & & & & Teste Z \\
\hline Limiar VA - $500 \mathrm{~Hz}$ & 52 & 46,6 & 47,5 & 13,1 & 20 & 75 & - \\
\hline Limiar VA - $2000 \mathrm{~Hz}$ & 52 & 38,2 & 35 & 15,8 & 20 & 70 & \\
\hline Diferença $500-2000(\mathrm{~Hz})$ & 52 & 8,5 & 10 & 13,1 & -30 & 35 & $<0,001^{*}$ \\
\hline Limiar VO - $500 \mathrm{~Hz}$ & 52 & 18,5 & 20 & 4,1 & 10 & 30 & \\
\hline Limiar VO - $2000 \mathrm{~Hz}$ & 52 & 23,8 & 25 & 6,5 & 10 & 30 & \\
\hline Diferença 500 X $2000(\mathrm{~Hz})$ & 52 & $-5,4$ & -5 & 6,5 & -20 & 10 & $<0,001^{*}$ \\
\hline
\end{tabular}

Legenda: $\mathrm{N}$ = número de orelhas; $\mathrm{VA}$ = via aérea; $\mathrm{VO}$ = via óssea; ${ }^{\star}$ diferença significativa 
situaram-se dentro da normalidade em ambos os grupos, com discreta elevação no grupo estudo (Tabela 2).

Nas Figuras 1 e 2, são apresentados exemplos dos resultados do registro dos limiares VA e VO obtidos em lactentes dos grupos controle e estudo.

Foi realizada uma análise da presença do gap aéreo-ósseo entre os grupos. Para isso, consideraram-se os valores médios do gap nas frequências de $500 \mathrm{~Hz}$ e $2000 \mathrm{~Hz}$. Houve presença de gap apenas no grupo estudo nas frequências de $500 \mathrm{~Hz}$ e $2000 \mathrm{~Hz}$, com diferença significativa, quando comparado ao controle, que não apresentou gap aéreo- ósseo (Tabela 3).

Vale ressaltar que a presença de gap aéreo-ósseo negativo, observada em alguns casos do grupo controle, ocorreu em razão de o registro de limiares eletrofisiológicos por via aérea

Tabela 2. Comparação dos valores médios dos limiares eletrofisiológicos em $500 \mathrm{~Hz}$ e $2000 \mathrm{~Hz}$ (dBnNA) por via aérea e via óssea entre os grupos

\begin{tabular}{|c|c|c|c|c|c|c|c|c|}
\hline Variável & Grupo & $\mathbf{N}$ & Média & Mediana & $\begin{array}{l}\text { Desvio } \\
\text { padrão }\end{array}$ & Mínimo & Máximo & $\begin{array}{l}\text { Valor de } \mathrm{p} \\
\text { Teste } \mathrm{Z}\end{array}$ \\
\hline Limiar em $500 \mathrm{~Hz}$ (VA) & GE & 52 & 46,6 & 47,5 & 13,1 & 20 & 75 & IC $(16,14 ; 23,84)$ \\
\hline Limiar em 2000 Hz (VA) & GE & 52 & 38,2 & 35,0 & 15,8 & 10 & 70 & IC $(12,07 ; 21,35)$ \\
\hline \multirow{2}{*}{ Limiar em 500 Hz (VO) } & GC & 82 & 16,3 & 20,0 & 5,1 & 5 & 25 & $0,008^{*}$ \\
\hline & GE & 52 & 18,5 & 20,0 & 4,1 & 10 & 30 & IC $(0,58 ; 3,78)$ \\
\hline Limiar em 2000 Hz (VO) & GE & 52 & 23,8 & 25,0 & 6,5 & 10 & 30 & IC $(0,15 ; 4,98)$ \\
\hline
\end{tabular}

Legenda: $\mathrm{N}$ = número de orelhas; $\mathrm{VA}$ = via aérea; $\mathrm{VO}$ = via óssea; $\mathrm{GC}$ = grupo controle; $\mathrm{GE}$ = grupo estudo; IC = Intervalo de confiança; ${ }^{*}$ diferença significativa

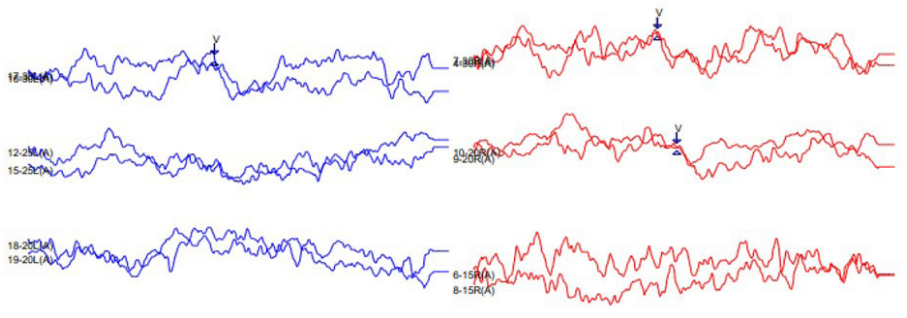

VA $2000 \mathrm{~Hz}$

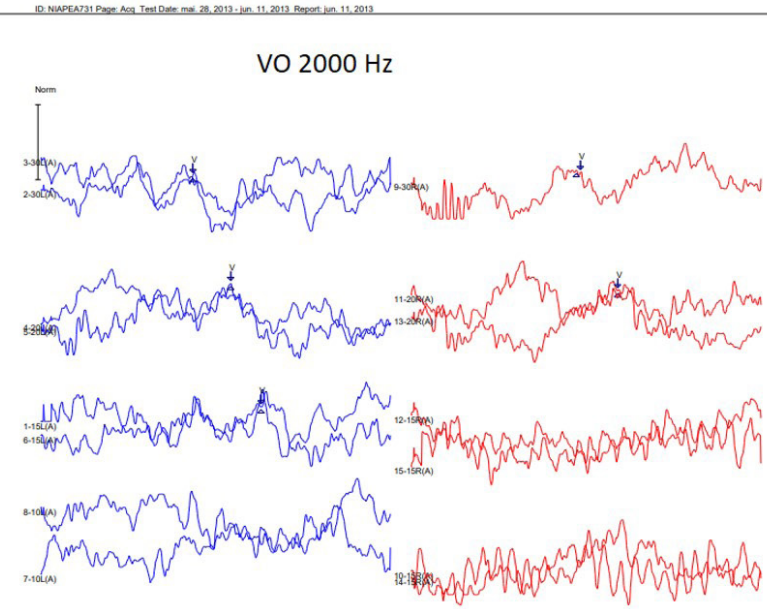

Figura 1. Registro de PEATE VA e VOna frequência de $2000 \mathrm{~Hz}$ em um lactente com audição normal Legenda: $V A=$ via aérea; $V O=$ via óssea
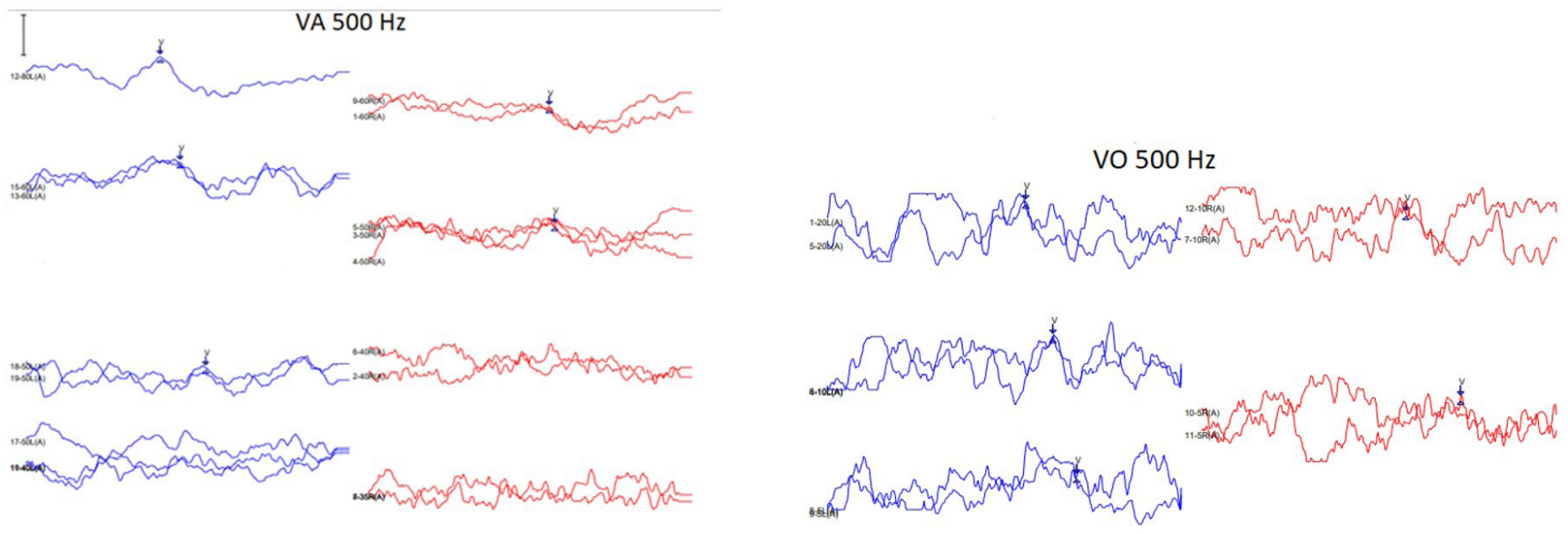

Figura 2. Registro de PEATE VA e VO na frequência de $500 \mathrm{~Hz}$ em um lactente com alteração condutiva Legenda: $\mathrm{VA}=$ via aérea; $\mathrm{VO}=$ via óssea 
situar-se acima dos limiares por via óssea, caracterizando ausência de gap.

Na população geral estudada (grupo estudo + grupo controle), analisou-se a relação entre a curva timpanométrica com duas variáveis: gap aéreo-ósseo e latência da onda I (Tabela 4). Houve maior ocorrência de curvas do tipo A nas orelhas com latência da onda I normal (95,3\%) e sem gap aéreo-ósseo (90,7\% em $500 \mathrm{~Hz}$ e $72,6 \%$ em $2000 \mathrm{~Hz}$ ), enquanto, nas orelhas com curva timpanométrica do tipo B, observou-se maior ocorrência de gap aéreo-ósseo e prolongamento da onda I $(85,4 \%)$. As curvas do tipo $\mathrm{C}$ estiveram presentes em 14,5\% das orelhas com latência da onda I aumentada e apenas 4,7\% delas com latência da onda I normal.

Foi observada associação significativa entre gap aéreo-ósseo e latência da onda I do PEATE clique. A latência da onda I aumentada associou-se à presença de gap, e a latência normal, à ausência de gap (Tabela 5).

\section{DISCUSSÃO}

Um dos fatores importantes a ser considerado na avaliação de lactentes é o tempo de realização do exame, principalmente quando ele é feito em sono natural. Neste estudo, o tempo médio para a pesquisa do limiar eletrofisiológico do PEATE por clique e do PEATE-FE (VA e VO), nas duas orelhas, foi de duas horas e 12 minutos, sendo que, em 68\% da amostra, a avaliação foi realizada em uma única sessão, independentemente do grupo. A duração do exame foi similar a um estudo da literatura ${ }^{(17)}$, porém, um pouco superior à média obtida em outro estudo ${ }^{(18)}$. Provavelmente, a maior duração do exame ocorreu por ausência de anestesia, pelo registro do tempo absoluto, sem considerar as pausas necessárias para garantir a condição do neonato e a pesquisa do limiar eletrofisiológico, invés do nível mínimo de resposta. Estudos ${ }^{(19,20)}$,que realizaram o PEATE em menor tempo, em geral, consideraram o nível mínimo de resposta, ou seja, a última resposta sem decrescer para visualizar a ausência da resposta, ou foram feitos com uso de anestesia. Apesar do tempo elevado, a avaliação completa foi realizada em todas as crianças e, em $68 \%$ da amostra, em única sessão. Tais resultados permitem considerar o registro do PEATE-FE por via aérea e óssea como um procedimento viável na prática clínica do serviço público de saúde, que nem sempre dispõe de sedação. $\mathrm{Na}$ prática clínica, uma alternativa para a redução do tempo da avaliação é considerar o nível mínimo de resposta invés do limiar eletrofisiológico.

O grupo estudo apresentou limiares eletrofisiológicos por via aérea aumentados e, por via óssea, normais, com gap aéreoósseo. O grupo controle demonstrou limiares eletrofisiológicos normais na condução aérea e óssea sem gap.

Neste estudo, os limiares médios obtidos por via aérea nos lactentes com comprometimento condutivo, situaram-se em $46,6 \mathrm{~dB}(500 \mathrm{~Hz})$ e $38,2 \mathrm{~dB}(2000 \mathrm{~Hz})$, similares a um estudo da literatura ${ }^{(21)}$,que encontrou limiares médios de $48 \mathrm{dBnNA}$

Tabela 3. Valores médios do gap aéreo - ósseo em $500 \mathrm{~Hz}$ e $2000 \mathrm{~Hz}$ por grupo e análise comparativa

\begin{tabular}{|c|c|c|c|c|c|c|c|c|}
\hline Variável & Grupo & $\mathbf{N}$ & Média & Mediana & $\begin{array}{l}\text { Desvio- } \\
\text { padrão }\end{array}$ & Mínimo & Máximo & Valor de $p$ \\
\hline \multirow[t]{2}{*}{ gap em $500 \mathrm{~Hz}$} & GC & 82 & 10,4 & 10 & 6,1 & -10 & 25 & $<0,001^{*}$ \\
\hline & GE & 52 & 28,3 & 30 & 13,2 & 0 & 60 & IC $(14,02 ; 21,79)$ \\
\hline gap em $2000 \mathrm{~Hz}$ & GE & 52 & 14,3 & 10 & 15,7 & -10 & 50 & IC $(9,42 ; 18,8)$ \\
\hline
\end{tabular}

Legenda: $\mathrm{N}$ = número de orelhas; $\mathrm{GC}$ = grupo controle; GE = grupo estudo; IC = Intervalo de confiança; ${ }^{\star}$ diferença significativa

Tabela 4. Comparação dos tipos de curva timpanométrica e presença de gap aéreo - ósseo e latência da onda I para o estímulo clique a 80 dBnNA ( $n=134$ orelhas)

\begin{tabular}{|c|c|c|c|c|c|}
\hline \multicolumn{6}{|c|}{ Tipo de curva timpanométrica } \\
\hline Gap & A & B & C & Total & $\begin{array}{c}\text { valor de } \mathbf{p} \\
\text { Teste } \mathbf{Z}\end{array}$ \\
\hline Com gap em $500 \mathrm{~Hz}$ & $23(33,3 \%)$ & $37(53,6 \%)$ & $9(13 \%)$ & $69(100 \%)$ & $<0,001$ \\
\hline Sem gap em $500 \mathrm{~Hz}$ & $59(90,7 \%)$ & $4(6,1 \%)$ & $2(3,0 \%)$ & $65(100 \%)$ & \\
\hline Total & 82 & 41 & 11 & 134 & \\
\hline Com gap em 2000 Hz & $4(14,8 \%)$ & $19(70,3 \%)$ & $4(14,8 \%)$ & $27(100 \%)$ & $<0,001$ \\
\hline Sem gap em 2000 Hz & $78(72,8 \%)$ & $22(20,5 \%)$ & $7(6,5 \%)$ & $107(100 \%)$ & \\
\hline Total & 82 & 41 & 11 & 134 & \\
\hline
\end{tabular}

Tabela 5. Associação entre presença e ausência de gap aéreo - ósseo e latência da onda I para o estímulo clique a 80 dBnNA

\begin{tabular}{|c|c|c|c|}
\hline & \multicolumn{2}{|c|}{ Latência onda I } & \multirow{2}{*}{ Total } \\
\hline & Alterada & Normal & \\
\hline Com gap $500 \mathrm{~Hz}(\geq 15)$ & $43(62,32 \%)$ & $26(37,68 \%)$ & $69(100 \%)$ \\
\hline Sem gap $500 \mathrm{~Hz}(<15)$ & $5(7,69 \%)$ & $60(92,31 \%)$ & $65(100 \%)$ \\
\hline Total Valor de $p<0,001$ & $48(35,82 \%)$ & $86(64,18 \%)$ & $134(100 \%)$ \\
\hline Com gap $2000 \mathrm{~Hz}(>15)$ & $21(75 \%)$ & $7(25 \%)$ & $28(100 \%)$ \\
\hline Sem gap $2000 \mathrm{~Hz}(<15)$ & 27 (25,48\%) & $79(74,52 \%)$ & $106(100 \%)$ \\
\hline Total Valor de $p<0,001$ & $48(35,82 \%)$ & $86(64,17 \%)$ & $134(100 \%)$ \\
\hline
\end{tabular}

Teste Z 
em $500 \mathrm{~Hz}$ e de $42,2 \mathrm{dBnNA}$ para $2000 \mathrm{~Hz}$. Vale ressaltar que os limiares em crianças com perda condutiva variam de acordo com o grau da perda e com a causa da alteração de orelha média.

A elevação dos limiares por via aérea no comprometimento condutivo já era esperada, uma vez que $80 \%$ das orelhas do grupo estudo apresentaram curva timpanométrica do tipo B. Nesses casos, a movimentação dos ossículos pode ser afetada pela presença de líquido mais espesso e viscoso, com maior conteúdo protéeico (glue-ear) ${ }^{(22)}$. Em $20 \%$ das orelhas, a curva timpanométrica obtida foi do tipo $\mathrm{C}$, caracterizando pressão negativa na orelha média, com retração de membrana timpânica e aumento de rigidez do sistema tímpano-ossicular ${ }^{(12)}$.

$\mathrm{Na}$ pesquisa dos limiares eletrofisiológicos por via óssea, o uso de mascaramento foi necessário, pois o equipamento disponível na instituição não dispunha de dois canais. Em equipamento de dois canais, a lateralidade da origem das respostas (qual cóclea está respondendo) pode ser determinada observando-se as assimetrias de latência e a amplitude dos registros da onda $\mathrm{V}$ ipsilaterais e contralaterais. Os registros das respostas ipsilaterais podem ser confirmados por presença de onda $\mathrm{V}$, com maior amplitude e menor latência em lactentes, em razão da atenuação interaural de até $25 \mathrm{~dB}$ nessa faixa etária ${ }^{(6)}$, inferindo-se que as respostas do PEATE-VO resultaram da estimulação da cóclea do mesmo lado. Os limiares por via óssea permaneceram dentro do padrão de normalidade nos dois grupos avaliados. Esse dado é relevante, pois indica haver a precisão diagnóstica, identificando tanto o grau, quanto o tipo de perda em lactentes que não respondem à audiometria convencional. Com a triagem auditiva neonatal, um significativo número de neonatos e lactentes é encaminhado para diagnóstico, e os resultados deste estudo confirmam a efetividade do uso desses procedimentos nessa população.

No grupo controle, os limiares médios obtidos por via aérea em $500 \mathrm{~Hz}(26,6 \mathrm{dBnNA})$ e $2000 \mathrm{~Hz}(21,5$ dBnNA) foram semelhantes aos obtidos em estudos na literatura ${ }^{(21,23)} \mathrm{e}$ discretamente superiores aos alcançados em outro estudo ${ }^{(18)}$. $\mathrm{Na}$ frequência de $2000 \mathrm{~Hz}$, os limiares foram melhores do que os obtidos em estudos brasileiros ${ }^{(20,24)}$ realizados com o mesmo equipamento. Essas diferenças devem-se ao fato de os estudos estabelecerem o nível mínimo de resposta, finalizando o exame ao atingir $30 \mathrm{dBnNA}$. Neste estudo, houve pesquisa do limiar eletrofisiológico de forma precisa, com decréscimo até a ausência de resposta. Por outro lado, um estudo internacional ${ }^{(21)}$ encontrou limiares eletrofisiológicos por via aérea ainda melhores, provavelmente pelo uso da sedação que reduz os artefatos e ruídos indesejados.

Em ambos os grupos, os limiares por via aérea em $500 \mathrm{~Hz}$ foram mais elevados em relação a $2000 \mathrm{~Hz}$. A justificativa para esse achado é a ausência de sedação, que prejudica a captação dos limiares, principalmente na frequência de $500 \mathrm{~Hz}$, mais afetada pela presença de ruídos.

Nos lactentes com comprometimento condutivo, a frequência de $500 \mathrm{~Hz}$ foi a mais acometida, tanto no neste estudo, quanto na literatura ${ }^{(21)}$. Provavelmente, esse fato ocorreu também em razão das alterações de rigidez sofridas na orelha média ${ }^{(1,22)}$. Efetivamente, limiares eletrofisiológicos mais elevados em 500 $\mathrm{Hz}$ por via aérea em crianças com e sem alterações condutivas já haviam sido encontrados na literatura ${ }^{(2,20,21,24)}$. Tal resultado também pode ser justificado pela dificuldade de obtenção e visualização da onda $\mathrm{V}$ em $500 \mathrm{~Hz}$, em fracas intensidades, visto que frequências baixas sofrem maior contaminação pelo ruído e precisam de mais tempo para atingir o ápice da cóclea e produzir respostas ${ }^{(25)}$. Dessa forma, a latência da onda $\mathrm{V}$ é mais longa e seu pico mais alargado, o que dificulta sua marcação. Uma forma de melhorar a relação sinal/ruído e possibilitar o registro dos limiares eletrofisiológicos em intensidades mais fracas é aumentar o número de estímulos por varredura para $3000^{(2)}$. Neste estudo, mesmo utilizando 3000 estímulos, os limiares em $500 \mathrm{~Hz}$ foram mais elevados em ambos os grupos. Esse fato era esperado, visto que os valores de correção sugeridos pelo protocolo canadense em 2012 consideram decréscimo de $15 \mathrm{~dB}$ em $500 \mathrm{~Hz}, 10 \mathrm{~dB}$ em $1000 \mathrm{~Hz}, 5 \mathrm{~dB}$ em 2000 e 0 em $4000 \mathrm{~Hz}$. Logo, os limiares estimados para o grupo controle estão situados entre 10 e $15 \mathrm{dBeNA}$ (limiares tonais estimados), caracterizando normalidade. No grupo estudo, após a correção, os valores permaneceram alterados em torno de $30 \mathrm{~dB}$ em $500 \mathrm{~Hz}$ e $25 \mathrm{~dB}$ em $2000 \mathrm{~Hz}$, confirmando a perda auditiva ascendente.

Os limiares eletrofisiológicos médios por via óssea estavam dentro dos padrões de normalidade em ambos os grupos, com melhores limiares em $500 \mathrm{~Hz}$, em relação à frequência de $2000 \mathrm{~Hz}$. Essa diferença pode estar relacionada à resposta de cada frequência pelo vibrador ósseo no crânio e suas características de transmissão ${ }^{(26)}$. Por esse motivo, sugere-se que a resposta da atividade da cóclea para frequência de $500 \mathrm{~Hz}$ seja melhor que na frequência de $2000 \mathrm{~Hz}$.

Em $500 \mathrm{~Hz}$, o limiar médio por via óssea foi $16,3 \mathrm{dBnNA}$ no grupo controle e 18,5 dBnNA no grupo estudo, similar aos obtidos na literatura ${ }^{(21,27)}$. Com a correção de $-5 \mathrm{~dB}^{(6)}$, os limiares estimados por via óssea estariam entre $10 \mathrm{~dB}$ no grupo controle e $15 \mathrm{~dB}$ no grupo estudo, caracterizando normalidade em ambos os grupos.

Para a frequência de $2000 \mathrm{~Hz}$, o limiar médio por via óssea foi 21,3 dBnNA no grupo controle e 23,8 dBnNA no grupo estudo, próximo ao encontrado na literatura ${ }^{(2)}$. Outros trabalhos ${ }^{(21,27)}$ apresentaram valores de limiares inferiores, em torno de $15 \mathrm{dBnNA}$. Uma justificativa para essa diferença é o tipo de equipamento utilizado na literatura, que é programado para facilitar a captação das respostas por um algoritmo em que é possível captar respostas ${ }^{(27)}$, mesmo com crianças agitadas e se movimentando, além do uso da fixação do vibrador por banda elástica, fatores que contribuem para a identificação de melhores limiares ${ }^{(21)}$.

Neste estudo, o fator de destaque foi a identificação da presença de gap aéreo-ósseo, o que facilita o diagnóstico do tipo de perda auditiva. No grupo de crianças com perda condutiva, houve a presença de gap aéreo-ósseo nas frequências de 500 e $2000 \mathrm{~Hz}$, com diferença significativa, em comparação ao grupo controle (Tabela 3). Na literatura consultada, havia apenas um trabalho ${ }^{(21)}$ contendo a análise do gap aéreo-ósseo entre crianças com audição normal e perda auditiva condutiva, na qual não foi observada gap em $500 \mathrm{~Hz}$ e em $2000 \mathrm{~Hz}$ nas crianças com audição normal, com diferenças de $15 \mathrm{~dB}$ e $5 \mathrm{~dB}$, dados similares aos obtidos neste estudo (diferenças de 10,4 e $0,2)$. Por outro lado, nas crianças com perda condutiva, houve presença de gap $(37 \mathrm{~dB}$ em $500 \mathrm{~Hz}$ e $26 \mathrm{~dB}$ em $2000 \mathrm{~Hz})$, ligeiramente superiores aos obtidos nesta pesquisa $(28,3 \mathrm{~dB}$ em $500 \mathrm{~Hz}$ e 14,3 dB em $2000 \mathrm{~Hz}$ ). Nesse mesmo estudo ${ }^{(21)}$, também foram encontrados gaps negativos, assim como neste trabalho, o que se justifica pela presença de limiares por via aérea melhores que por via óssea, principalmente no grupo controle, caracterizando ausência de gap.

É importante ressaltar que a maior ocorrência de gaps foi obtida na frequência de $500 \mathrm{~Hz}$, assim como os maiores gaps. Dessa forma, para otimizar o tempo do exame em neonatos, a 
frequência de $500 \mathrm{~Hz}$ deve ser a escolhida para a pesquisa da via óssea. De fato, na prática clínica, a configuração ascendente das perdas condutivas, em decorrência do aumento de rigidez, propicia maior gap nas baixas frequências.

Neste estudo, a pesquisa das curvas timpanométricas, utilizando sonda de $1000 \mathrm{~Hz}$ nos lactentes com idade inferior a 6 meses, identificou a presença de alteração condutiva, visto que houve associação entre curva alterada e presença de gap aéreo-ósseo (Tabela 4). De fato, estudos ${ }^{(28,29)}$ demonstram que lactentes com idade inferior a 6 ou 9 meses e sem resposta nas emissões podem apresentar um timpanograma normal, com tom de teste de $226 \mathrm{~Hz}$, mesmo quando há alteração condutiva, caracterizando baixa sensibilidade na identificação de problemas leves de orelha média. Na época deste estudo, a recomendação do uso da sonda de $1000 \mathrm{~Hz}$ era para lactentes com idade inferior a 6 meses. A literatura mais recente recomenda a sonda de 1 $000 \mathrm{~Hz}$ em crianças de até 9 meses, faixa etária que apresenta maior correlação com a avaliação otorrinolaringológica e com o resultado das emissões otoacústicas e maior sensibilidade para identificar alterações de orelha média ${ }^{(4,28,29)}$.

Perante o exposto, o estudo considera os achados da literatura, pois, no grupo de lactentes com ausência de gap, $90,7 \%$ e $72,6 \%$, respectivamente para as frequências de 500 e $2000 \mathrm{~Hz}$, apresentaram curva timpanométrica do tipo A. Além disso, houve predomínio de curvas tipo A $(95,3 \%)$ nas orelhas com latência da onda I normal, e maior ocorrência da curva tipo B $(85,4 \%)$ nas orelhas com prolongamento da latência da onda I, com evidência de associação entre o tipo de curva e a latência da onda I (Tabela 4).

Dessa forma, a presença de gap aéreo-ósseo no PEATE nas frequências de 500 e $2000 \mathrm{~Hz}$ pode ser considerada como forte indicativo de comprometimento condutivo. Além disso, a associação significativa entre a presença de gap aéreo-ósseo e o aumento da latência da onda I, observada neste trabalho (Tabela 5), também foi referida na literatura ${ }^{(23) .}$

Entretanto, neste estudo, das 86 orelhas com latência da onda I normal, quatro (4,6\%) tinham alteração condutiva, ou seja, a latência da onda I não foi suficiente para identificar $100 \%$ das perdas condutivas. (Tabela 5). De fato, alguns estudos relatam que a onda I pode não ser um bom preditor para identificar perdas condutivas $^{(2,30)}$, assim como revelar o tamanho do gap $^{(21)}$. Por outro lado, alguns lactentes com perda condutiva apresentaram aumento da latência da onda I sem presença de gap, em uma das frequências pesquisadas ( $500 \mathrm{ou} 2000 \mathrm{~Hz}$ ), pois a criança poderia apresentar ausência de gap em $2000 \mathrm{~Hz}$ com gap em $500 \mathrm{~Hz}$ (Tabela 5). Além disso, a latência aumentada da onda I, em alguns lactentes, pode estar associada ao comprometimento condutivo decorrente da curva $\mathrm{C}$, que nem sempre é acompanhado de presença de gap aéreo-ósseo.

As associações da timpanometria alterada e da latência prolongada da onda I, com a presença de gap aéreo ósseo no PEATE, reforçam a importância clínica da utilização da combinação do PEATE VA e VO por frequência específica como uma alternativa viável e eficaz no diagnóstico das perdas auditivas condutivas em lactentes.

\section{CONCLUSÃO}

Os resultados deste estudo permitem considerar o registro do PEATE-FE por via óssea um procedimento viável na prática clínica de um serviço público de saúde, que nem sempre dispõe de sedação.

As associações da timpanometria alterada e da latência prolongada da onda I, com a presença de gap aéreo-ósseo no PEATE, reforçam a importância clínica da utilização da combinação do PEATE VA e VO por frequência específica como uma alternativa viável e eficaz no diagnóstico das perdas auditivas condutivas em lactentes.

\section{AGRADECIMENTOS}

Agradecemos a todos os familiares e crianças que participaram do estudo e à fonoaudióloga Dra Flávia Ribeiro, pelo auxílio desde a coleta de dados até a elaboração e revisão do artigo.

\section{REFERÊNCIAS}

1. Homøe P, Heidemann CH, Damoiseaux RA, Lailach S, Lieu JEC, Phillips JS, et al. Panel 5: impact of otitis media on quality of life and development. Int J Pediatr Otorhinolaryngol. 2020 Mar;130(Supl. 1):109837. http://dx.doi.org/10.1016/j.jporl.2019.109837. PMid:31883704.

2. Stapells DR. Frequency-specific evoked potential audiometry in infants. In: Seewald RC, editor. A sound foundation through early amplification. Chicago: Phonak; 2000. p. 13-31.

3. Baljić I, Walger M. Objective frequency-specific measurement of hearing threshold using narrow-band chirp stimuli with level-adaptive simultaneous masking. HNO. 2019 Nov;67(11):843-54. PMid:31197424.

4. Joint Committee on Infant Hearing. Year 2019 position statement: principles and guidelines for early hearing detection and intervention programs. J Early Hear Detect Interv. 2019;4:1-44. http://dx.doi. org/10.15142/fptk-b748.

5. Birkent ÖF, Karlıdağ T, Başar F, Yalçın Ş, Kaygusuz İ, Keleș E, et al. Evaluation of the relationship between the air-bone gap and prolonged ABR latencies in mixed-type hearing loss. J Int Adv Otol. 2017;13(1):8892. http://dx.doi.org/10.5152/iao.2016.1731. PMid:27819648.

6. BCEHP: British Columbia Early Hearing Program. Diagnostic audiology protocol. Vancouver; 2012.

7. Ontario Infant Hearing Program. Protocol for auditory brainstem response: based audiological assessment (ABRA) [Internet]. Toronto; 2018 [citado em 2021 Maio 14]. Disponível em: https://www.uwo.ca/ nca/pdfs/clinical_protocols/2018.01\%20ABRA\%20Protocol_Oct $\% 20$ 31.pdf

8. BSA: British Society of Audiology. Recommended procedure Auditory Brainstem Response (ABR) testing in babies [Internet]. 2019 [citado em 2021 Maio 14]. Disponível em: https://www.thebsa.org.uk/wpcontent/uploads/2019/04/Recommended-Procedure-for-ABR-Testingin-Babies-FINAL-Feb-2019.pdf

9. Lightfoot G. Sloping ABR baselines and the ECG myogenic artefact. Int J Audiol. 2017;56(8):612-6. http://dx.doi.org/10.1080/14992027 .2017.1313463. PMid:28415901.

10. Lau R, Small SA. Effective masking levels for bone conduction auditory brainstem response stimuli in infants and adults with normal hearing. Ear Hear. 2021;42(2):443-55. http://dx.doi.org/10.1097/ AUD.0000000000000947. PMid:32925305.

11. Seo YJ, Kwak C, Kim S, Park YA, Park KH, Han W. Update on bone-conduction auditory brainstem responses: a review. J Audiol 
Otol. 2018;22(2):53-8. http://dx.doi.org/10.7874/jao.2017.00346. PMid:29471611.

12. Jerger J. Clinical experience with impedance audiometry. Arch Otolaryngol. 1970;92(4):311-24. http://dx.doi.org/10.1001/ archotol.1970.04310040005002. PMid:5455571.

13. Gorga MP, Worthington DW, Reiland JK, Beauchaine KA, Goldgar DE. Some comparisons between auditory brain stem responses thresholds, latencies and pure tone audiogram. Ear Hear. 1985;6(2):105-12. http:// dx.doi.org/10.1097/00003446-198503000-00008. PMid:3996784 .

14. Stuart A, Nelson HM. The effect of bone vibrator coupling method on the neonate auditory brainstem response. Int J Audiol. 2019;58(6):339-44. http://dx.doi.org/10.1080/14992027.2019.1578426. PMid:30849923.

15. Stuart A, Dorothy HM. Neonate auditory brainstem response repeatability with controlled force gauge bone-conducted stimulus delivery. Int J Audiol. 2018;57(1):76-80. http://dx.doi.org/10.1080/14992027.2017 .1374567. PMid:28918681.

16. Kramer SJ. Frequency specific auditory brainstem responses to bone conducted stimuli. Audiology. 1992;31(2):61-71. http://dx.doi. org/10.3109/00206099209072902. PMid:1610314.

17. Polonenko MJ, Maddox RK. The parallel auditory brainstem response. Trends Hear. 2019;23:2331216519871395. http://dx.doi. org/10.1177/2331216519871395. PMid:31516096.

18. Sininger YS, Hunter LL, Hayes D, Roush PA, Uhler KM. Evaluation of speed and accuracy of next-generation auditory steady state response and auditory brainstem response audiometry in children with normal hearing and hearing loss. Ear Hear. 2018;39(6):1207-23. http://dx.doi. org/10.1097/AUD.0000000000000580. PMid:29624540.

19. François M, Teissier N, Barthod G, Nasra Y. Sedation for children 2 to 5 years of age undergoing auditory brainstem response and auditory steady state responses recordings. Int J Audiol. 2012;51(4):282-6. http://dx.doi.org/10.3109/14992027.2011.601469. PMid:21936745.

20. Porto MAA, Azevedo MF, Gil D. Potenciais evocados auditivos em lactentes pré-termo e a termo. Rev Bras Otorrinolaringol. 2011;77:622-7.

21. Vander Werff KR, Prieve BA, Georgantas LM. Infant air and bone conduction tone burst auditory brain stem responses for classification of hearing loss and the relationship to behavioral thresholds. Ear Hear. 2009;30(3):350-68. http://dx.doi.org/10.1097/AUD.0b013e31819f3145. PMid:19322084.
22. Kaytez SK, Ocal R, Yumusak N, Celik H, Arslan N, Ibas M. Effect of probiotics in experimental otitis media with effusion. Int J Pediatr Otorhinolaryngol. 2020;132:109922. http://dx.doi.org/10.1016/j. ijporl.2020.109922. PMid:32036169.

23. Michel F, Jørgensen KF. Comparison of threshold estimation in infants with hearing loss or normal hearing using auditory steady-state response evoked by narrow band CE-chirps and auditory brainstem response evoked by tone pips. Int J Audiol. 2017;56(2):99-105. http://dx.doi. org/10.1080/14992027.2016.1234719. PMid:27715342.

24. Sleifer P, Didoné DD, Keppeler IB, Bueno CD, Riesgo RDS. Air and bone conduction frequency-specific auditory brainstem response in children with agenesis of the external auditory canal. Int Arch Otorhinolaryngol. 2017;21(4):318-22. http://dx.doi.org/10.1055/s-0037-1598243. PMid:29018492.

25. Hall JW 3rd. Update on auditory evoked responses: evidence-based ABR protocol for infant hearing assessment. Audiology Online [Internet]; 24 jan 2017 [citado em 2021 Maio 14]. Disponível em: http://www.audiologyonline.com

26. Yang EY, Rupert AL, Moushegian G. A developmental study of bone conduction auditory brainstem responses in infants. Ear Hear. 1987;8(4):244-51. http://dx.doi.org/10.1097/00003446-19870800000009. PMid:3653538.

27. Elsayed AM, Hunter LL, Keefe DH, Feeney MP, Brown DK, Meinzen-Derr JK, et al. Air and bone conduction click and tone-burst auditory brainstem thresholds using kalman adaptive processing in nonsedated normal-hearing infants. Ear Hear. 2015;36(4):471-81. http://dx.doi.org/10.1097/ AUD.0000000000000155. PMid:25738572.

28. Yerraguntla K, Kaur R, Ravi R. A preliminary attempt to profile tympanometric measures in infants using high frequency probe tones. Indian J Otolaryngol Head Neck Surg. 2018;70(2):188-93. http:// dx.doi.org/10.1007/s12070-016-1004-2. PMid:29977839.

29. AAA: American Academy of Audiology. Clinical guidance document assessment of hearing in infants and young children. Reston; 2020.

30. Mackersie CL, Stapells DR. Auditory brainstem response Wave I prediction of conductive component in infants and young children. Am J Audiol. 1994;3(2):52-8. http://dx.doi.org/10.1044/1059-0889.0302.52. PMid:26661607. 